\title{
Consequences of repeated discovery and benign neglect of non-interaction of waves (NIW)
}

ChandraSekhar Roychoudhuri

ChandraSekhar Roychoudhuri, "Consequences of repeated discovery and benign neglect of non-interaction of waves (NIW)," Proc. SPIE 10452, 14th Conference on Education and Training in Optics and Photonics: ETOP 2017, 1045215 (16 August 2017); doi: 10.1117/12.2266216

Event: 14th Conference on Education and Training in Optics and Photonics, ETOP 2017, 2017, Hangzhou, China 


\title{
Consequences of repeated discovery and benign neglect of Non- Interaction of Waves (NIW)
}

\author{
ChandraSekhar Roychoudhuri ${ }^{\mathrm{a} *}$ \\ ${ }^{a}$ Department of physics, University of Connecticut, Storrs, CT, USA 06269-3046 \\ *Chandra.Roychoudhuri@uconn.edu
}

\begin{abstract}
This paper presents the historical background behind the repeated discovery and repeated ignoring of the generic important property of all propagating waves, the Non-Interaction of Waves (NIW). The focus will be on the implications of NIW in most of the major optical phenomena with brief hints of importance. We argue that the prevailing postulate of wave-particle duality becomes unnecessary, once we accept NIW. Semi-classical model of treating light-matter interactions should be the preferred approach since the quantumness actually arises from within the structure of the energy levels (bands) in materials. Waves, and wave equations, do not support bullet-like propagation. We follow the historical trend starting from the tenth century physicist Alhazen, to the seventeenth century Newton and Huygens, then to the nineteenth century Young and Fresnel. Then we jump to twentieth century physicists Planck, Einstein, Bose, Dirac and Feynman. Had we recognized and appreciated NIW property of waves from the time of Alhazen, the evolutionary history of physics would have been dramatically different from what we have today. The prevailing dominance of the postulate of wave-particle duality is keeping us confused from seeking out actual reality; and hence, we should abandon this concept and search out better models. The paper demonstrates that NIW provides us with a platform for deeper understanding of the nature of EM waves that we have missed; it is not just semantics.
\end{abstract}

Keywords: Non-Interaction of Waves; NIW; History of Non-Interaction of Waves; Implications of NonInteraction of Waves in modern physics; Linear Superposition Principle; Quadratic Superposition Effect; Physics theories are always incomplete

\section{INTRODUCTION}

Many critical thinkers have recognized the generic property of waves that we are calling, Non-Interaction of Waves (NIW). However, standard textbooks do not explicitly articulated this NIW property. The implication is that the wave amplitudes, by themselves, can interfere with each other and re-organize their mutual field energy distributions, directly generating fringes. This implied meaning undermines the reality that the energy is always measured as some physical transformation in some detector that is directly proportional to the square of the sum of all the superposed amplitude stimulations the detector experiences. The mathematical structure of the Superposition Principle is essentially correct; so it is validated by experimental data. However, we ignore the necessity of deeper introspection regarding what are the physical processes that makes light waves observable to us. We unconsciously ignore the fact that we never see light. We only perceive (interpret) the light induced physical transformations experienced by detectors, whether our retinal pixels, or modern camera pixels. Thus, the Superposition Principle (SP), expressed mathematically as the linear sum of more than one physically superposed wave amplitudes, is not directly observable. It is the square modulus of the sum total dipolar stimulation induced on a detector, is the observable Superposition Effect (SE). This distinction, apparent from the NIW property, would lead us to develop a causally self-consistent model of a "photon". Most likely, it would be a discrete packet of energy at the moment of emission (atomic transition), which immediately evolves as an exponentially decaying classical wave packet. This would bridge the classical and quantum gap for electromagnetic waves. 


\section{BRIEF HISTORY RECOGNITION \& NEGLECT OF NIW [1]}

\subsection{Ibn al-Haytham (Alhazhen), a major physicist ( 965 - 1040) of the Arab world}
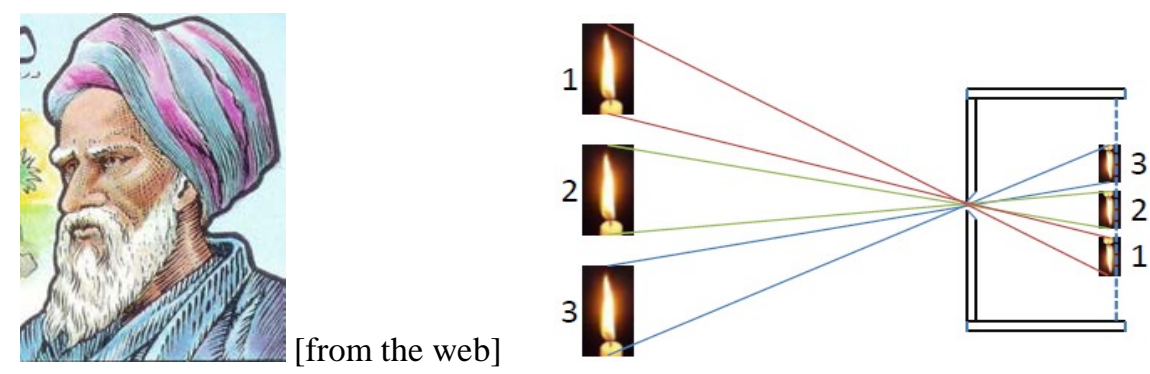

Figure 2.1. Left Image: Huygens’ portrait [Google Image]. Right Sketch: A sketch depicting his possible experiemental arrangement to discover NIW [Fig.2.1b in ref.2].

Al-Haytham was a well-known physicist of the $11^{\text {th }}$ century with major contributions in basic optics [3]. He carried out a remarkable simple experiment with a pinhole camera (camera-obscura) and a set of candles. He observed that neither blocking or nor un-blocking some of the candles have any influence on the images of the remaining candles. Therefore, he correctly concluded that light, as some energetic signals, cross through each other unperturbed (without any interaction) through the pinhole to form the inverted images of the candle flames. As an enquiring physicist, he also underscored that he could not figure out the real physical nature of light, which remains true even today [2]. Serious physics in the Western world started with Galileo (1564-1642) and Newton (1642 -1726). Thus, Alhazhen was the first scientist to recognize formally the Non-Interaction of Waves (NIW); even though he could not articulate that way since the wave concept for light, as propagating waves of some tension field would develop some 600 years later.

\subsection{Christian Huygens (1629 - 1695)}
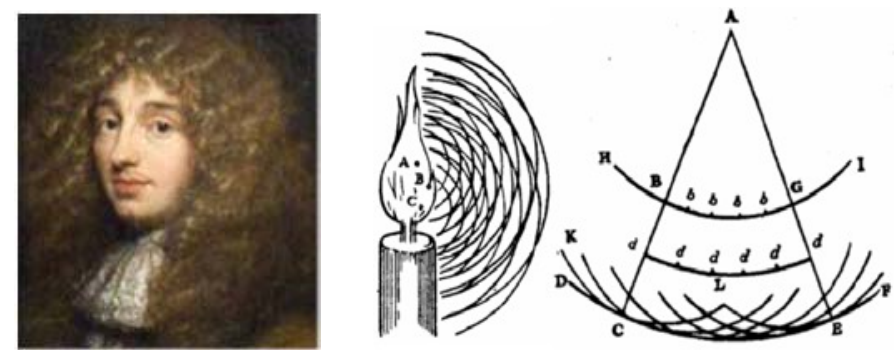

Figure 2.2. Left Image: Huygens portrait [Google Image]. Right Sketch: Diffractive propagation of light wave as non-interacting secondary spherical wavelets. [4]

Huygens was a life-long hands-on multi-talented scientist. He was the first one to define the nature of light as a form of excitation of some space pervading "ether field". He proposed that every point on a wave front behaves as a new source point for the generation of a secondary spherical wavelet. This postulate was originally presented in 1678 at the Paris Academy of Sciences and was published as a book in 1690 [4]. All the Huygens wavelets keep expanding, generating the evolving diffraction fields, later known as near-field (Fresnel diffraction) and far-field (Fraunhofer diffraction) patterns. Huygens explicitly mentioned in his book that these secondary wavelets evolve through each other unperturbed by the overlapping mutual presence and without re-arranging the energy distribution of each other. This is NonInteraction of Waves (NIW); although he did not use this phrase. Huygens postulate also implicates that the quiescent ether tension filed is the real source of energy and hence light can propagate perpetually through the entire cosmic space without any aid from the original light emitting source. Conceptually, this is very similar to sound waves leveraging the pressure tension field of the air for its propagation; water waves leveraging the surface tension of the water surface and string-waves propagating over the mechanical tension field held by the stretched string. During 1817, Fresnel was the first one to give a mathematical integral structure to Huygens' Principle as literal sum of infinite number of secondary wavelets, now known as Huygens-Fresnel Diffraction Integral (HF) [5]. Note that the very foundation of physical optics 
relies on this Huygens-Fresnel diffraction integral. The mathematical justification of the HF integral was given by the Maxwell wave equation that accepts any linear combination (summation) of any set of harmonic oscillations.

Till today, the sustained and continuous growth of optical science and engineering (Physical Optics, Fourier Optics, Image processing, etc., etc.) rely on this HF integral (and/or Maxwell's wave equation). No optical engineer uses any mathematics that resembles literal propagation of indivisible light quanta; even though most of us give lip service to this misleading concept. Optical scientists and engineers can appreciate this further by imagining how the mathematical foundation of light propagation through complex optical system would look like, if by magic, the knowledge of the Huygens Principle and HF integral vanish from all the publications and all of our minds! Notice, in contrast, at the high end of physics, we are already postulating Dark Energy and Dark Matter to accommodate the measured velocity distribution of stars in galaxies, which cannot be modeled either by Newtonian or by the Einsteinian theories of Gravity [6]. The Dark Energy is effectively embedded in Huygens postulate since he assumed that the "ether field" is a real energetic physical tension field that aids the perpetual propagation of light waves through the entire cosmic space.

\subsection{Newton (1642-1726).}

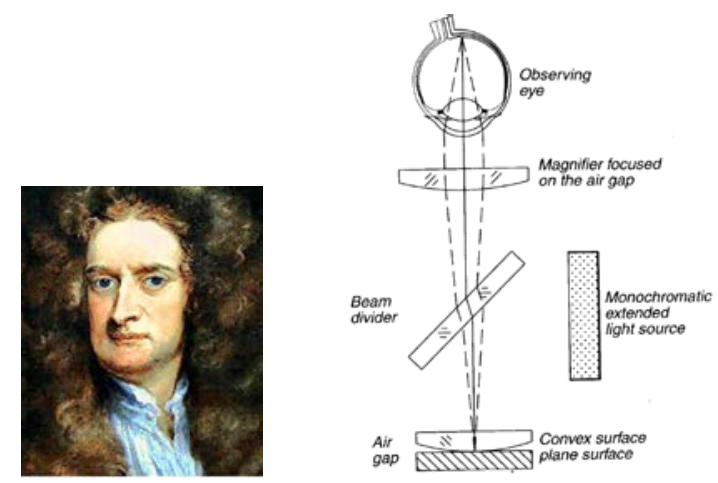

Figure 2.3. Left Image: Newton's portrait [Google Image]. Right Sketch: Newton's interferoemeter to measure the radius of curvature of his plano-convex lens polished to construct his telescopr [see Fig.1.1 in ref.7].

Newton, the major giant in formulating the foundation of physics, was a contrarian to Huygens' wave concept. He proposed alternative corpuscular theory in 1704 in his Optiks book [8]. Throughout 1700, under the prestigious weight of Newton, people tried to accept the corpuscular theory; but the model failed even to explain the foundational laws of refraction from a lighter to a denser medium and the progress in optics remained rather slow. However, Newton was fully aware that his corpuscular light executed periodic harmonic oscillation and the Superposition Effect of light (interference), based on his experience in inventing and using Newton's Interferometer [7] to measure the radius of his plano-convex lenses he was polishing to construct telescopes. Most likely, he was using Bunsen burner and Sodium salt to generate quasi-monochromatic light for his interferometer. Being well aware of the law of conservation of energy, he realized that the discrete Na-atoms in the flame could emit only discrete packets of light energy. This is the most probable reason why he used the word corpuscular (particle-like) rather than light as literally particles.

It was Huygens and Newton, who initiated the debate of wave-particle duality during late 1600. However, both of them were aware that the key reason behind this conceptual duality was due to the prevailing ignorance behind the fundamental knowledge about the physical nature of light; and it was not because nature itself harbors duality. However, what was accepted as our ignorance during 1600 until the beginning of 1900, was converted into a new assumed knowledge. The author still believes that the duality still represents our ignorance, not a new knowledge. We still do not know what constitute photons and electrons [2]. 


\subsection{Thomas Young (1773 -1829)}
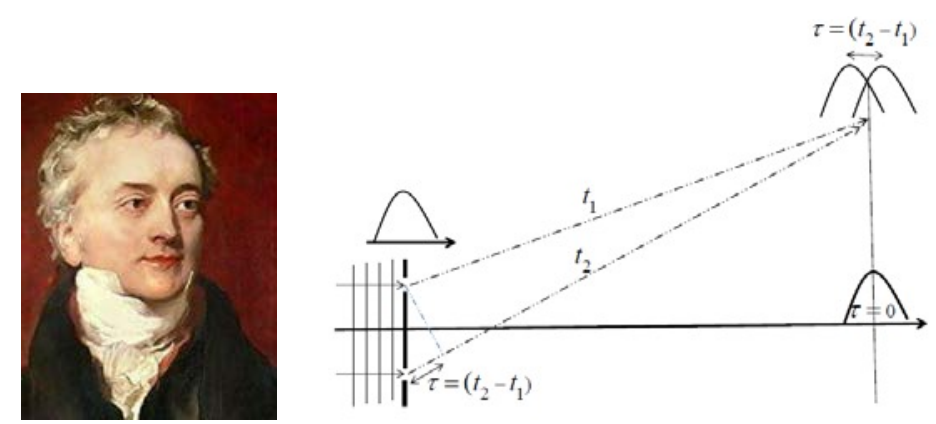

Figure 2.4. Left Image: Portrait of Thomas Young [Google Image]. Right Sketch: A sketch of the well-known double-slit interference (diffraction) experiment. It underscores differential diffractive time delays (diffractive pulse broadening), varying continuously from the on-axis to the off-axis cases [see Fig.10.3 in ref.2].

It was during 1801 when Thomas Young succeeded in vindicating Huygens's wave theory by demonstrating his, now famous, double slit Superposition Effect (interference). During the entire century of 1800, the field of classical optical science and engineering evolved and matured dramatically. Young, most likely, did not carefully read either Huygens' presentation at the French Academy or his 1690 book where non-interaction of the wavelets was explicitly mentioned. Young used the term interference, without recognizing that the Superposition Effect is a quadratic energy transfer process from all the superposed field amplitudes to our retinal "pixels"! Co-propagation or cross-propagation of wave amplitudes through the same volume cannot generate re-distribution of energy (observable fringes) in the absence of quadratically responding detecting entities. Even the modern classical and quantum physicists have been perpetuating the same conceptual mistake; even though they all represent the final registered fringes by some detector array as the square modulus of the sum of all the wave amplitudes. What have we been ignoring systematically? It is the detector that carries out the physical process of taking the square modulus of the superposed wave amplitudes before they can absorb the proportionate amount of energy from all the superposed stimulating fields.

\subsection{Augustin-Jean Fresnel (1788 - 1827).}
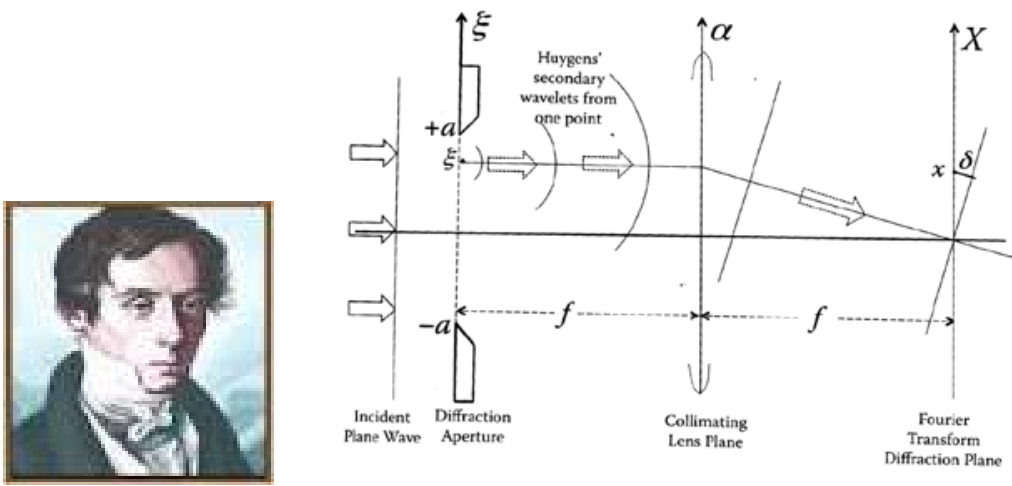

Figure 2.5. Left Image: Portrait of Fresnel (from Google Image). Right Sketch: The evolution of one of many secondary wavelets (quadratic phase curvature) in the near field (between the slit and the first lens, the "alpha"-plane). They remain unobstructed by each other (note that only one spherical wavelet is drawn). After the "Fourier transform" focusing lens, all the secondary wavelets become tilted plane waves (linear phase). The simultaneous superposed stimulation of all these plane wavelets on a detector array will give the quadratic energy transfer corresponding to the Fraunhofer (far-field-like) intensity variation due to the single slit [see Fig.4.5 in ref.2].

In 1816, Fresnel gave the mathematical structure to Huygens Principle, as literally the summation (integral) of an infinite number of secondary spherical wavelets, $[\exp (i k r) / r]$, emanating out of every point on the aperture. The inclination factor, $\cos \theta$, in Eq.1 makes the amplitudes of the secondary wavelets die out rapidly for large angular divergence [5]. Of course, the registered energy distribution (the diffraction pattern) can be determined only by a detector array at any 
forward plane we place by executing the square modulus operation on all the secondary wavelets $\left|U\left(P_{\circ}\right)\right|^{2}$ on that forward plane (see Eq.2). Note that $\chi(\lambda)$ is the detector's wavelength (or frequency) dependent dipolar response property to the oscillating E-field providing the stimulation before energy transfer. The propagating wave amplitudes keep evolving unperturbed through each other until they hit a detector array at any forward plane. This is Non-Interaction of Waves (NIW). This is already built into the amplitude diffraction Eq.1.

$$
\begin{gathered}
U\left(P_{0}\right)=\frac{-i}{\lambda} \iint_{\Sigma} U\left(P_{1}\right) \frac{\exp \left(i k r_{01}\right)}{r_{01}} \cos \theta d s \\
\left|U\left(P_{0}\right)\right|_{\text {Detector }}^{2}=\left|\frac{-i}{\lambda} \iint_{\Sigma} \chi(\lambda) U\left(P_{1}\right) \frac{\exp \left(i k r_{01}\right)}{r_{01}} \cos \theta d s\right|^{2}=\frac{\chi^{2}}{\lambda^{2}}\left|\iint_{\Sigma} U\left(P_{1}\right) \frac{\exp \left(i k r_{01}\right)}{r_{01}} \cos \theta d s\right|^{2}
\end{gathered}
$$

The physical implication of the summation (integration) operation in Eq.1 only implies the co-existence and copropagation of wave amplitudes, as long as the medium is linear and any quadratic energy exchange (transfer) process is absent. However, in Eq.2, the integral implies that the detecting dipole is physically summing and executing the resultant simultaneous stimulations, $\chi(\lambda) U\left(P_{1}\right)$, induced by all the locally present fields $U\left(P_{1}\right)$. When the optical frequency of the field is resonant with the intrinsic quantum property of the detector; then the detector executes the square-modulus energy transfer process, which makes it release the photoelectron. These details behind physical detection processes were not known in the early days; although it was known that the field energy is proportional to the square of the amplitude. People accepted the interpretation of Eq.1 as the Superposition Principle as if the wave amplitudes interfere by themselves. However, physics never found any interaction forces among different light wave amplitudes in the absence of interacting materials. For a given monochromatic wave, the polarizability $\chi(\lambda)$ is a constant. Therefore, by the accepted mathematical rule, $\chi^{2}(\lambda)$ can be taken out of the integral and the square modulus operations (see Eq.2). It then appears merely a detector constant. The resultant mathematical expression still validates the measured data. Thus, this mathematical rule, devoid of detector stimulation, implies that the Superposition Principle is effectively observable as interference between wave amplitudes. In practice, only the detector's physical transformation through the square modulus step, which we call the Superposition Effect (photoelectron transfer), is observable. This is more than semantics. For centuries, we have missed recognizing the NIW property of waves, which has deep implications in all of physics [see Ch.10-12 in 2].

However, because of the "measurable correctness" of the diffraction theory, the growth of the field of optical science and engineering bloomed during the entire century of 1800 following Young's double slit interference experiment and Fresnel's mathematical formulation of the Huygens Principle.

\subsection{Max Planck (1858 -1947)}

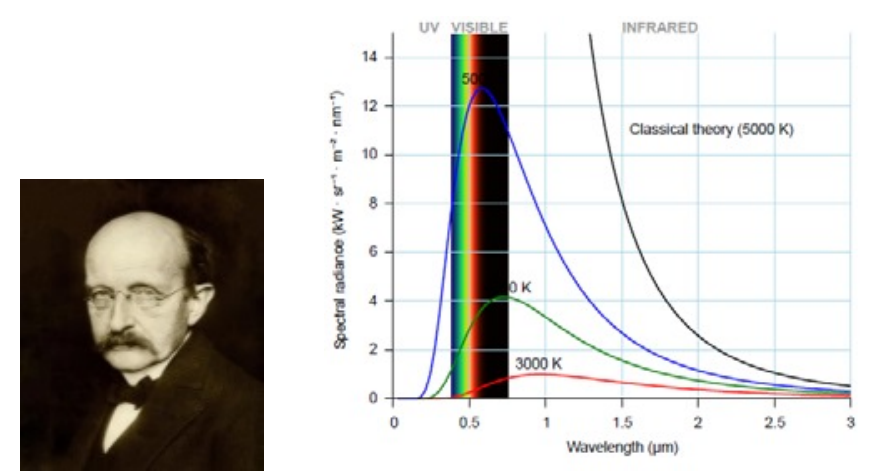

Figure 2.6. Left Image: Planck's portrait [from Google Image]. Right Graph: Planck's formula correctly predicting Blackbody cavity energy distribution with wavelength under equilibrium at any particular temperature [from Google Image]. 
Then in, 1900 Planck actually validated Newton's hunch that atoms can release only discrete quantities of energy from the standpoint of conservation of energy. However, the issues were much more complex than just corpuscular vs. wave. To match the experimentally measured energy emission curve from Blackbody cavity, Planck was compelled in 1900 to usher in the very foundation of quantum mechanics. Atoms and molecules not only emit and absorb energy in discrete amounts (as Newton surmised), but the discrete energy packets are uniquely dictated by the optical frequency as $h v$ (not the wavelength, which changes from medium to medium for the same frequency). However, Planck still underscored in his 1913 book [9] during the detailed derivation behind the Planck's Equation that light always propagates unperturbed (NIW-property re-recognized) while spreading diffractively through each other. He had presented strong causal arguments. Had light propagated as discrete packets while interacting with each other, steady state Blackbody equation would have required such an interaction term to achieve the thermal equilibrium! Therefore, Planck remained a life-long proponent of light as classical wave packets. (i) During the moment of emission, it is a transient discrete energy packet (or transient photon) of energy $h v$ that evolves as a transformed classical wave packet of the ether (cosmic medium). (ii) During absorption, atoms or molecules individually functions as a quantum cup [2]. They fill up their quantum cups with only the necessary quantity of energy $h v$ out of all the frequency resonant and inphase wave packets passing by them. The fractional amount of energy taken from each wave packet is determined by our standard mathematical superposition equation, the square modulus of the sum of all the in-phase wave packet amplitudes $\left|\sum_{n} \chi(v) E_{n}(v)\right|^{2}$ stimulating it simultaneously. Surprisingly, this correct mathematical representation of the energy transfer process, first starting with the linear Superposition Principle of summing all the simultaneously present amplitudes, does not dictate that only one lucky "indivisible light quanta”, out of many optical beams, can successfully impregnate the targeted atom or molecule. The theory of QM does not require that the energy donor, to facilitate an upward quantum transition, must also have a matching quantum of energy. The necessary quantum of energy can be donated even through classical kinetic collisions between atoms and molecules. That is why Boltzmann's population distribution relation permeates the temperature dependent quantum excitation phenomena.

\subsection{Albert Einstein (1879 -1955)}
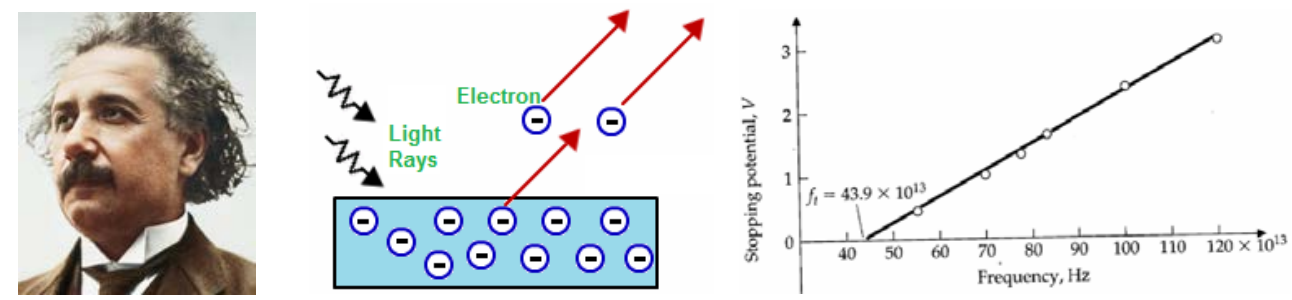

Figure 2.7. Left Image: Portrait of Einstein [from Google Image]. Middle Cartoon: A cartoon] represents the release of quantum mechanically bound electrons in some material after being energized by light wave packets [Google Image]. Right Plot: Einstein's brilliance was that he found some quantumness in the experiemntal curve of photoelectric effect. Had he assigned this quantuness to the bound electrons in materials; he would have succeded in formulating quantum mechanics with his own logic some twenty years erarlier [see Fig.4.8 in ref.10].

Einstein was the first one to propose the concept of "indivisble light quanta" through his 1905 paper on photoelectric effect. This was in spite of his knowledge of Plancks publications and talks since the beginning of 1900. Planck never believed in "indivisble light quanta", not during early 1900 and not during the rest of his life. Eisntein simply employed the Measutrable Data Modeling Epistemology (MDM-E) [see Ch.12 in ref.2] and gave us the following equation to perfectly model the above curve:

$$
h v=\phi_{\text {work fn. }}+(1 / 2) m v_{e l .}^{2}
$$

Interestingly, Einstein was known to have said a few months before his death that he had been brooding over the concept the question, "What are light quanta", for over fifty years without finiding a satisfactory answer. Therefore, the author was inspired to explore whether semi-classical model can be applied to photoelectric effect. In fact, many well known people have already been promoting this semi-classical modles [11-15]. I am presenting here a simple approach.

We now know that electrons are always bound quantum mechanically in all materials, whether in sharp levels, as in atoms and simple molecules, or in wide bands, as in material in solid states. Further, during the days of Einstein, the concept of generation and manipulation of single photons did not exist. In his days, optical sources were always 
producing incoherent light beams containing simply innumerable wave packetsp (or Einstein's photons). Further, we need to map the physical process of light-matter interaction (the stimulation process) before elcetrons can be released out of their bound states. Elementary stimulation of the electron holding dipole complex is expressed as:

$$
\psi=\chi\left(v_{q}\right) E\left(v_{q}\right)
$$

Since there are always multiple wave packets that can simultaneously stimulate the same dipole complex, we should employ Interaction Process Mapping Epistemology (IPM-E) and write:

$$
\psi_{\text {res. }}=\sum_{q} \chi\left(v_{q}\right) E\left(v_{q}\right)
$$

However, we know that the release of a bound electron requires a quadratic energy absorption process:

$$
\left|\psi_{\text {res. }}\right|^{2}=\left|\sum_{q} \chi\left(v_{q}\right) E\left(v_{q}\right)\right|^{2} \propto h v_{q}
$$

A single data point cannot generate a re-verifiabkle curve. Only ensemble average can generate a data-curve:

$$
\left\langle\left|\psi_{\text {res. }}\right|^{2}\right\rangle=\left\langle\left|\sum_{q} \chi\left(v_{q}\right) E\left(v_{q}\right)\right|^{2}\right\rangle \propto\left\langle h v_{q}\right\rangle=\left\langle\phi_{\text {work fn. }}+(1 / 2) m v_{\text {el. }}^{2}\right\rangle
$$

Eq.7 shows the connectivity between Einstein's original photoelectric Eq.3, derived by using just the data modeling epistemology, and our physical process mapping epistemology. Let us now go a step further. Had Eisntein used the interaction process mapping epistemology (IPM-E), he could have noted that for a very narrow band of incident frequency, the polarizability factor will be a constant and we can get the mistaken concept that wave amplitudes can sum themselves even in the absence of interacting materials (also compare with the right-hand-side of Eq.2):

$$
\left|\psi_{\text {res. }}\right|^{2}=\left|\sum_{q} \chi\left(v_{q}\right) E\left(v_{q}\right)\right|^{2}=\chi^{2}\left|\sum_{q} E\left(v_{q}\right)\right|^{2}
$$

A careful comparison of the above two summation expressions is worth underscoring again. The square-modulus expression in the middle of Eq.8 clearly implies that the detecting molecular complex is executing the simultaneous dipolar stimulations induced by all the superposed fields on it, effectively carrying out the physical superposition effcet. Howver, using the allowed mathematical rule that one can take out a constant out of the two consecutive mathematical operators, $\sum$ and ||$^{2}$, we can generate the mistaken physical interpretation that the wave amplitudes are summing

themselves, $\sum_{q} E\left(v_{q}\right)$. This is still the dominant belief, that wave amplitudes directly interfere (interact)! The author believes that by ignoring the active role of light detectors, we are continuing our mistaken belief for centuries, that light interferes directly with light, while ignoring the enormous implications of NIW in all branches of physics.

\subsection{Satyendra N. Bose (1894-1974)}

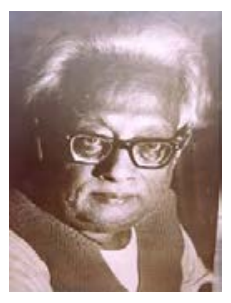

Figurer 2.8. Portrait of Bose [Google Image].

Bose was the first one to claim in his 1924 publication [16-18] that he had derived the Planck's Radiation Law using a fully quantum mechanical concept. His elegant derivation has eventually given rise to the famous Bose-Eisntein Statistics, a field that in recent decades, has garnered several Nobel Prizes. Bose's mathematics identified that the photon number in his phase-sapce box reamins conserved (unchnged) even though they are confined inside the "box". The 
author believes that Bose's mathematical observation, no change in the photon number, has also implicated that photons (light waves) do not interact with each other; and hence preserves the photon number as conserved.

\subsection{Luis de Broglie (1892 -1987)}
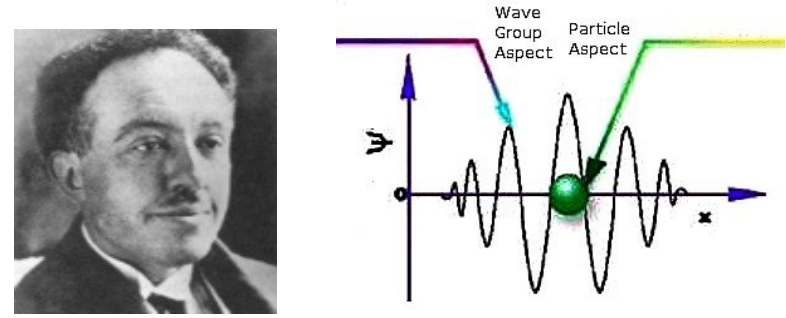

Figure 2.9. Left Image: Portrait of de Broglie [from Google Image]. Right Cartoon: A depiction of wave-particle duality formalized by de Broglie's research and development of the concept of Pilot Wave [from Google Image].

The author believes that the postulate, wave-particle duality, still represnts our deper ignorance as to what light waves and particles are. This was also the belief of Newton and Huygens, who actullay originated this concept of duality (corpuscular vs. waves) almost 400 hundred years ago. However, de Broglie's excellent research work on "Pilot Waves" during 1926 triggered the formalization of our deeper ignorance, as to what really constitutes light (photons) and particles, into a new knowledge of “wave-particle duality”!! However, the superposition effects produced by particles does not require either the particles to be plane waves, or to be guided by Pilot Waves. In the section 2.11, we have presented our strictly causal approach to explain superposition effects due to partilces as registered by detecting particles without the need for Pilot Waves. We just need to introduce the postulate that particles possess velocity-dependent internal harmonic oscillations, the phase of which gives rise to phase-sensitive superposition effects, like propagating waves do. The model is very similar to that due to superpsoition effect generated, point by point, by detecting molecules while absorbing energy from all the stimulating particles incident on them.

\subsection{P. A. M. Dirac (1902-1984)}
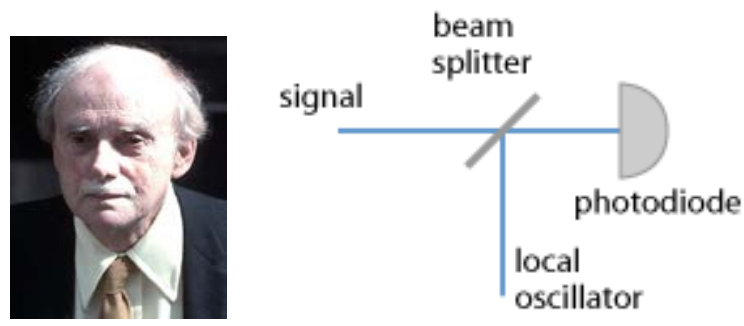

Figure 2.10. Left Image: Portrait of Dirac [from Google Image]. Right Cartoon: Baisc schematics for heterodyne detection of optical signals by superpsoing light beams of two different frequencies from two different sources [from Google Image]. This is a well established technique in Laser radar and LIDAR technologies. Different "photons" from different sources do generate Superpsoition Effcets as wave packets.

Dirac is known for his famous declaration that a photon can interfere only with itself and different photon do not interfere. The last phrase clearly indicates that Dirac mathematically found the generic property of waves, that they do not interact (interfere) by themselves in the linear domain. However, most likely, in defference to the prevailing, but wrong, classical belief that light wave amplitudes do interfere; Dirac gave us a non-causal postulate that an indivisble and stable "photon" can make itself appear or disappear based upon the mechanical state of interferometric alignments; even though no force of interaction between photons in the linear domain were theorized by Dirac.

Very successful and matured technologies behind Laser radar and LIDAR decisively prove that light beams from independent sources do generate Superposition Effects; irrespective of whether we call them photon or not. Dirac has also given the mathematical foundation of quantizing the Elctromagnetic Waves [19] consisting of discrete photons as Fourier modes of the vacuum. However, Dirac' photons cannot be localized by superposition of many Fourier modes because of the NIW property, the main theme of this paper. 

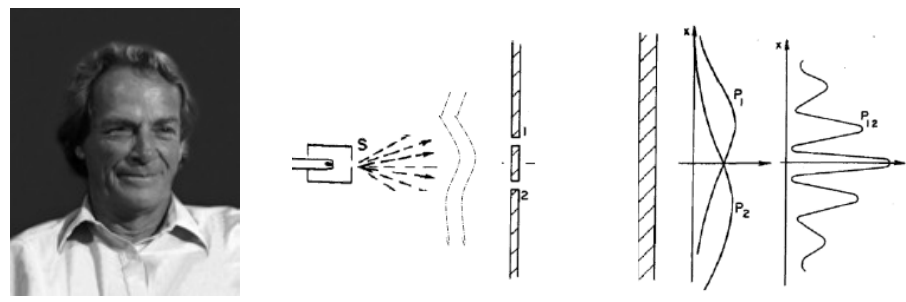

Figure 2.11.1. Left Image: Portrait of Feynman from the web. Right Sketch: Double-slit Superposition Effect with particles, adapted from the book, Feynman Lectures.
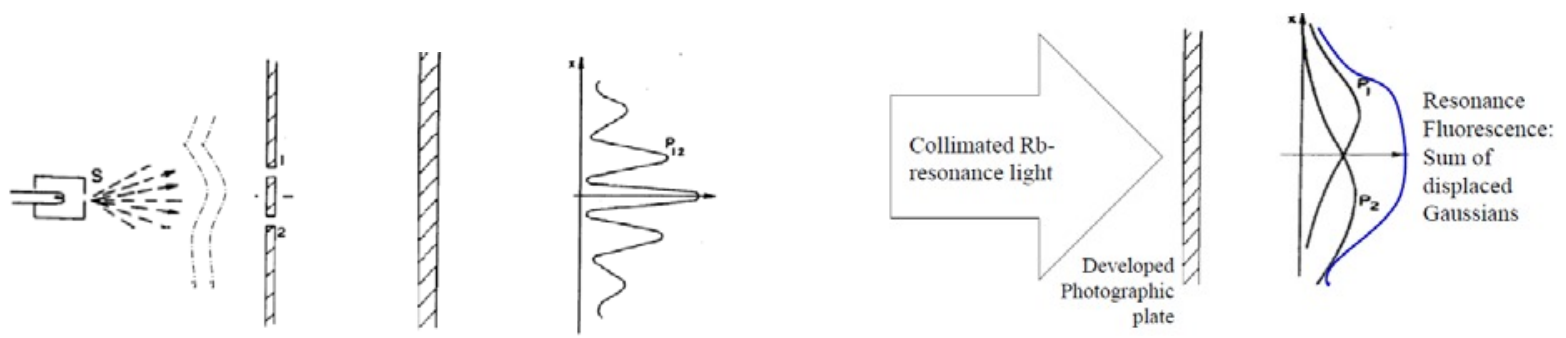

Figure 2.11.2. Proposed repetition of Feynman's thought experiment with Rb-atomic beam, instead of electron beam (as presented in his book, Feynman Lectures). Left Sketch: A thick photographic emulsion does the recording of the superposition effect. Its development will show standard double-slit cosine fringes under the single-slit sinc-squared envelope function (not shown). The fringes represent the superposition effect generated by the Rb-atoms due to their simultaneous arrival as in-phase and out-of-phase stimulations of the Ag-Halide crystallites. Right Sketch: The developed photograph is illuminated by a collimated Rb-resonance $780 \mathrm{~nm}$ laser beam. The intensity of the fluorescence would show the sum of two Gaussian curves representing physical arrival distribution of the actual Rb-atoms.

Feynman had famously said that nobody understands quantum mechanics; so keep computing to get the right results. The mathematical formalism of Quantum Mechanics (QM) has been with us for over 90 years. If it is working, it must have more realities built into it than we have understood, so far. The reason we still do not understand why QM works, is because we are not trying to explore the invisible physical interaction processes that give rise to the measurable data. The key weakness of QM is that its formalism does not provide us with any explicit guidance in visualizing the invisible interaction processes of the micro world [see Ch.12 in 2]. However, the key purpose of physics is to understand and visualize all interaction processes that are behind the persistent evolution. This is a required condition towards our successful Geo-engineering and Cosmo-engineering that would be required to assure the continued evolution of biological lives forever.

The continued success of our mathematical equations (theories) is based upon our standard procedure of equating measurable effects with the postulated causes to model nature. We also know from our long historical observations that all working theories pave the way to develop better and newer theories. Therefore, instead of assuming that QM is the final theory for the micro world; we should proactively use violations of causality, locality, etc., as nature provided cues to frame newer questions leading to better theories. That has been the history of evolution of all scientific theories. Keeping alive the spirit of persistent enquiry by proactively discovering the weakness or incompleteness of a working theory should be the key art behind excellent teaching, not by permanent conformation to the working theory by contriving newer and newer postulates to accommodate the weaknesses.

The author is presenting the double-slit experiment with the proposed collimated Rb-beam, as presented in Fig.2.11.2 [2] to explore a causal approach to explain the observed Superposition Effects with beams of particles. The key enquiring question is as follows. Can a stable elementary particle like an electron, or an atom, carry more than one dynamic properties for the same physical parameter at the same moment? Can a stable elementary particle veer its trajectory without the direct aid of some force introduced by some external agency and keep them from arriving at precisely the pre-ordained (by superposition equation) dark fringe locations? These causal questions are now suppressed by the ad hoc postulates like non-causality, non-locality, entanglement, delayed choice, etc., etc.

Therefore, the author is proposing a possible causal postulates. The dark fringes are due to normal unperturbed and simultaneous arrival of pairs of particles that are out of phase in their internal kinetic-velocity determined oscillation 
frequency $f$ [see Ch.11 in ref.2]. These mutually out of phase particles neutralizes the delivery-potential of their energy to the detecting molecule. The detecting molecule remains unexcited. It cannot absorb energy from the arriving particles. Therefore, the corresponding physical locations appear as "dark". The dark fringes are not due to non-arrival of the stimulating particles. Again, this is also built into our superposition equation, if we scrutinize carefully.

$$
\begin{aligned}
D(\tau)= & \left|\chi a_{1} r e^{i \pi} e^{i 2 \pi f(t+\tau)}+\chi a_{2} e^{i 2 \pi f t}\right|^{2}=\chi^{2}\left|a_{1} r e^{i 2 \pi f(t+\tau)}+a_{2} e^{i 2 \pi f t}\right|^{2} \\
= & \chi^{2}\left[\left(a_{1}^{2}+a_{2}^{2}\right)-2 a_{1} a_{2} \cos 2 \pi f \tau\right] \\
= & 2 \chi^{2} a^{2}[1-\cos 2 \pi f \tau] ; \text { only when } a_{1}=a_{2}=a \\
& \Uparrow \text { Linear dipolar polarizability of the detector molecules. }
\end{aligned}
$$

The detector's detectivity is $D(\tau)$, and $\chi$ is the linear dipolar polarizability of detecting molecules. The relative delay $\tau$ in the arrival of two particles from the two separate slits is defined in the first sketch of Fig.2.11.3. The indivisible particles are bound to have different trajectories with different times of arrivals on any particular detecting particle starting from the two different slits. The simultaneous amplitude and phase stimulations experienced by any detecting particle, $\chi a_{1} \exp [2 \pi f(t+\tau)]$ and $\chi a_{1} \exp [2 \pi f t]$, are induced by the simultaneous arrival of two different particles carrying their oscillatory characteristics, $a_{1} \exp [2 \pi f(t+\tau)]$ and $a_{1} \exp [2 \pi f t]$. The number of ensemble averaged successful excitations would vary as $\cos 2 \pi f \tau$ (over the DC-bias).

It is obvious that a single data point cannot provide us with the cosine fringe curve. We must record a very large number of statistically random events to make a visible (measurable) curve. One of the apparent weaknesses of this proposed postulate is also its strength. The random temporal array of particles cannot systematically arrive as pairs at the different sites of detecting molecules. In other words, this model predicts that there would always be un-paired particles at the dark-fringe sites that will stimulate the detecting molecule and create a detected event. In other words, according to this model the dark fringes-locations in particle diffraction pattern can never have perfect zeros, or show perfect visibility fringes. In fact, this is the case indicated by the double-slit neutron diffraction pattern registered by Zeilinger [20]. We show this in the in the middle diagram of Fig.2.11.3 below, where we also present the corresponding optical diffraction pattern with perfect visibility, for comparison (extreme right). Optical perfect visibility is obtainable because optical waves diffractively spread out as per Huygens postulate of secondary wavelets. Stable individual particles do not spread out like waves do. They follow the exact trajectory determined by the scattering phenomenon, or real physical forces, when applied. [Interestingly, Feynman's most well know contribution is the mathematical employment of "all possible paths" for particle interactions; was derived by taking analogy from Huygens' perpetual spreading of secondary wavelets.]
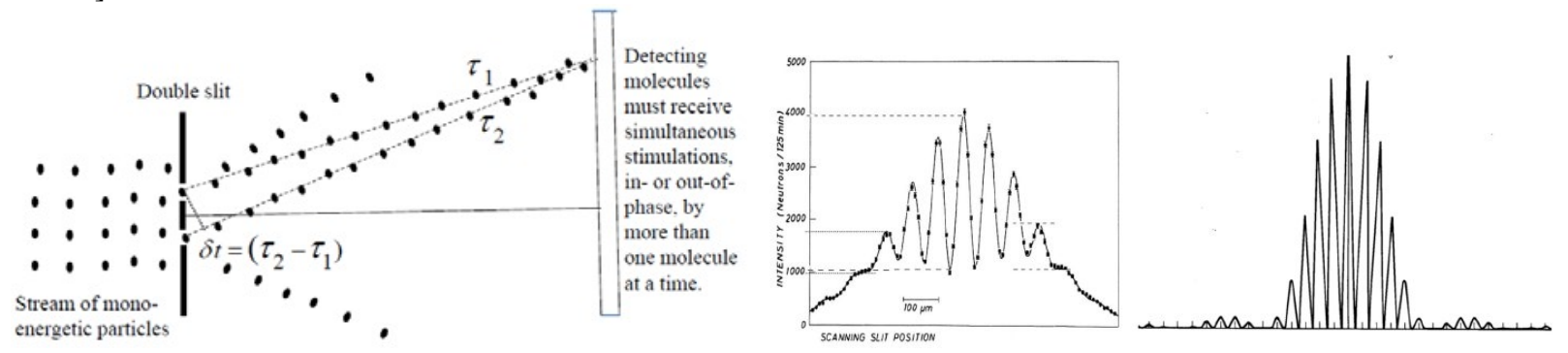

Figure 2.11.3. Left Sketch: Individual particles are scattered by each one of the two slits with Gaussian angular distribution (see again the Fig. 2.11.1 and 2.11.2). Right Curves: Double-slit diffraction patterns are compared for the cases of indivisible particles and divisible optical waves (Huygens Principle). Author's prediction is that, for particle beam diffraction, the dark fringe-locations will always have non-zero detection counts [see Fig.11.5 \& 11.6 in ref.2 ], [20].

We have already recognized earlier that the superposition of light beam amplitudes, by themselves, do not produce energy re-distribution (the NIW property). In fact, they pass through themselves without any mutual interaction in the linear domain. Unlike light beams, "rigid" particles cannot pass through each other. They get scattered by each other and/or interact with each other based, on the available (operational) mutual force. However, in the presence of a stimulate-able detecting molecule, the stimulating factors, $a_{1} \exp [2 \pi f(t+\tau)]$ and $a_{1} \exp [2 \pi f t]$, are not interacting 
mutually to repel themselves from "dark fringe" locations. They are stimulating the detecting molecule simultaneously, but out of phase; the effect is nulled. Simultaneous in-phase arrival generates real physical stimulation and hence energy transfer from two or more particles to one detecting molecule; which give rise to particle superposition effect. To rephrase, when the phases of a pair of simultaneously arriving particles differ by $180^{\circ}$ (opposite phase), they cannot stimulate the detecting molecule and no transfer any energy can take place. This is the dark fringe location. When the "diffracted" particles are stable Rb atoms, as has been proposed in Fig.2.11.3, they remain embedded in the same "dark fringe" locations without being able to generate any local excitations (energy exchange). Since the scattering of neutral particles by a slit would be close to Gaussian, we have predicted above that the sum total distribution of the embedded $\mathrm{Rb}$ atoms in the detecting photographic emulsion would be the sum of two slightly displaced Gaussian curves, as predicted above. Further, the particles carrying the phase sensitive oscillatory signals, $a_{1} \exp [2 \pi f(t+\tau)]$ and

$a_{1} \exp [2 \pi f t]$, are not themselves physically entangled as they are propagating independently without any force of interaction between them. However, they are entangled only locally, in the limited sense that they are simultaneously interacting with the detecting particle and jointly delivering energy to it. The physical range of this interaction is also quite small (the dipolar stimulation of Ag-halide molecule), which would be in the domain of multiples of atomic diameter.

Let us also recognize that the Ag-Halide molecules, in the crystallites of the detecting photographic plate, are bound quantum mechanically. However, the energy transfer process here is purely classical, kinetic collision between stationary Ag-Halide molecules and kinetic Rb-atoms, albeit possessing oscillatory phases. However, the darkening of the photographic plate is discrete, only because the Ag-Halide molecules are discrete and they are also embedded in discrete micro crystallites. This is why all developed photographic plates, when sufficiently enlarged, will always appear grainy irrespective of whether they are exposed by beams of discrete particles or wave packets of light; or, whether the beams are very weak or very strong.

Mathematically, the Eq.9 applies equally well had we used light beams through an appropriate double-slit aperture using the same photographic plate as the detector array. A long exposure (time integrated ensemble average) will register fringes as $2 a_{1} a_{2} \cos 2 \pi f \tau$ over the DC bias $\left(a_{1}^{2}+a_{2}^{2}\right)$ when $a_{1} \neq a_{2}$. Notice that the registered signal (energy transfer) is proportional to the strength of both the beams, $a_{1}$ and $a_{2}$. Therefore, our standard mathematics does not support the claim that the detector accepts energy only from one of the beams and as a discrete packet. This assumption contradicts our successful mathematical formalism, which have been framed with causality, equating cause and effect, as the foundation.

\subsection{Author's 1975-76 Experiments}

During 1975-76, the author carried out a series of experiments [21,22] using a tilted Fabry-Perot (a pair of parallel beam splitters) and a He-Ne laser beam, as depicted in Fig.2.12. During this period, the author was not aware of the simple and elegant experiment of Alhazen. Some details of this experiment are given as captions within the Fig.2.12. The fan of independent and parallel He-Ne beams, produced by the FP, was focused on a ground glass by a lens. Its polished flat surface was set towards the FP and the ground surface towards the right, the direction of observation. The flat surface reflected back the convergent fan of laser beams as a divergent set of beams, as required by the standard law of reflection. The Poynting vector of each of the beam guides its propagation away from the glass plate independent of the fact that they were reflected out of the same focal region on the glass plate where they were physically superposed. Thus, physical superposition alone does not create Superposition Effect. This is Non-Interaction of waves (NIW). In reality, this should have been obvious to us since ancient times when we formulated the laws of reflection. Otherwise, the same mirror segment could not have been creating separate images of innumerable separate objects in front of the mirror. However, we have not been paying attention!

The same silica molecules, as on the flat side, when grounded up to lumpy surface with small sizes comparable to the incident wavelength, they are scattering out the incident light as multitudes of Huygens secondary wavelets dictated by the local phases of the incident waves. In any particular far field plane, they generate the usual speckle pattern due to superposition of all the randomly phased wavelets without any macro superposition fringes. The macro Superposition Effect, however, would be evident when the scatter plate surface is re-imaged on an energy detection plane (retina of the eyes, CCD camera, etc.) where the energy absorption is proportional to the square modulus of the sum of all the local E- 
vectors. In the dark fringe minima locations, the resultant E-vector amplitudes would be minimum, causing a minimum of energy absorption. In the bright fringe maxima locations, the resultant E-vector amplitudes would maximum, causing a maximum of energy absorption. The basic physics has already been known from theory of light scattering [5], speckle pattern generation by laser beams; scatter plate interferometry [7], etc.

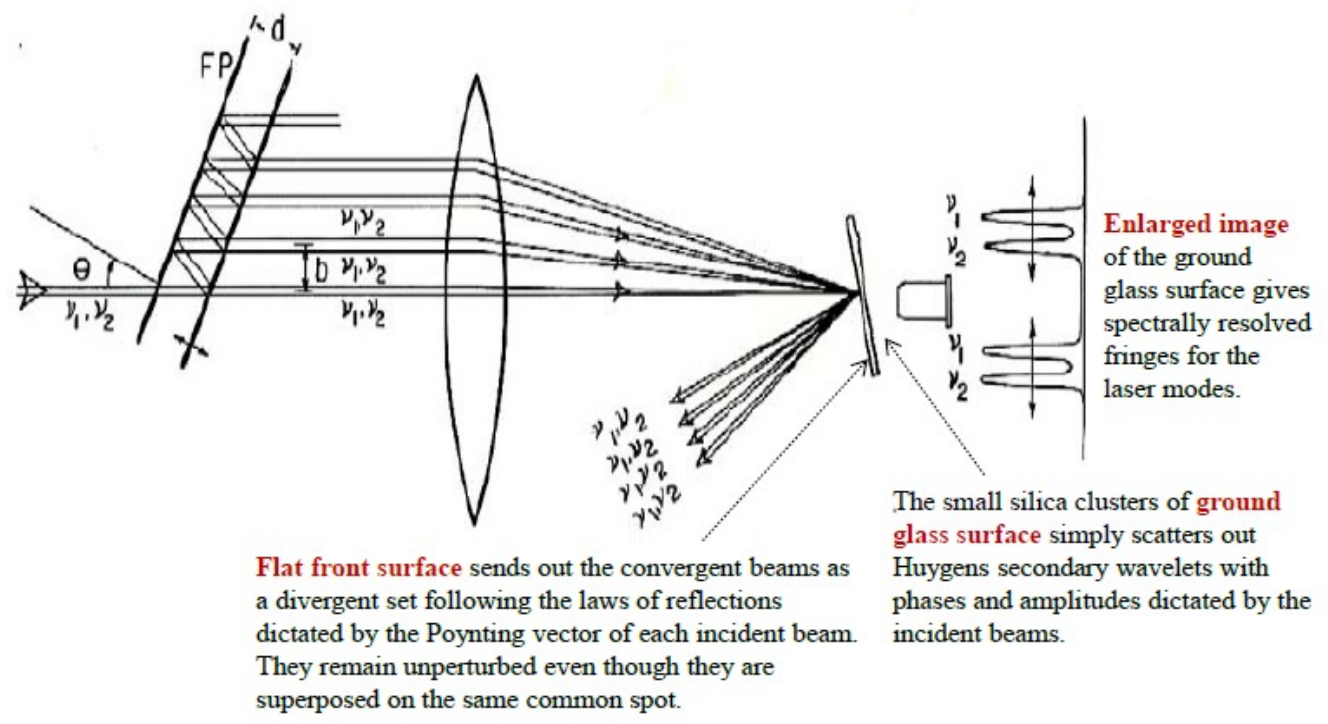

Figure 2.12. A tilted Fabry-Perot generates a fan of spatially independent set of parallel beams. They are focused on a polished glass plate. The sketch and the captions describe 1975-76 experiments carried by the author to appreciate, for the first time, that Non-Interaction of Waves (NIW) is a very important property of propagating waves [see Fig.2.2 in ref.2 \&22]

Generation of superposition (interference) fringes does not require the quantum postulate of "indivisible light quanta", or quantum detectors. We have known that well before the advent of Quantum Mechanics. However, somehow, we have not been paying attention! One can fully explain Superposition Effect by semi classical model even when the detectors are quantum mechanical, releasing quantum mechanically bound electrons in materials. The quantum, mechanical detectors behave as quantum cups to absorb the necessary quantity of energy, dictated by the quantum dipolar resonant frequency. EM filed does not require quantization. Physics behind light-matter interaction processes become much better that way. The quantumness resides in the materials.

\section{CONCLUSION}

Explicit recognition of NIW helps us remove a good number of contradictions in and conflicting interpretations of a good number of classical and quantum optical phenomena [2]. Huygens' postulate of non-interacting secondary wavelet has in it the built-in proposition that the cosmic space consists of a stationary Complex Tension Filed (CTF). This is the reason why the star light can perpetually propagate across the entire cosmic volume with the same constant velocity determined by its intrinsic electric and magnetic tension properties, $\varepsilon_{0}^{-1} \& \mu_{0}$. This was later mathematically found by Maxwell, $c^{2}=\left(\varepsilon_{0}^{-1} / \mu_{0}\right)$, corroborating the mathematical structure of the velocity of propagating waves in many other tension fields. Thus, NIW has deep implications in Quantum and Cosmological Physics [see Ch.10-12 in the book of ref.2]

It is rather surprising why we have not been incorporating this critical NIW property of waves in our interpretations of superposition effects! It was obvious to Alhazen from a simple experiment a thousand years ago while imaging lit candles with a camera-obscura [3]. Huygens formally postulated NIW almost four hundred years ago [4]. The author believes that it is due to our over emphasis on MDM-E (Measurable Data Modeling Epistemology); rather than empowering the intrinsic strength of the very practical MDM-E by adding the system engineering philosophy of IPM-E (Interaction Process Mapping Epistemology) [see Ch.12 in the book, ref.2]. 


\section{REFERENCES}

[1] The summary of the content of this Section 2 has been used by the author as a pamphlet to promote the yearly Technical Event, "The Nature of light: What are photons?" at the SPIE Photonics West Conferences since (2010).

[2] C. Roychoudhuri; many papers related to this book are available for download from: http://www.natureoflight.org/CP/; [Causal Physics: Photon Model by Non-Interaction of Waves], Taylor \& Francis, Hardcopy (2014), Paperback (2017).

[3] V. Ronchi, see p.53 in [The Nature of Light], Harvard University Press (1970).

[4] C. Huygens, Traité de la Lumière (drafted in 1678; but published in Leyden by Van der Aa, 1690), translated by S. P. Thompson as [Treatise on Light], London, Macmillan, 1912; See http://www.gutenberg.org/ebooks/14725 for free download from Project Gutenberg edition (2005). C. Huygens (1690), translated by Silvanus P. Thompson (1912), Treatise on Light, London: Macmillan, 1912; Project Gutenberg edition, 2005; Errata, 2016.

[5] M. Born and E. Wolf, [Principles of Optics], Cambridge U. Press (1999).

[6] R. Panek [The 4 Percent Universe: Dark Matter, Dark Energy, and the Race to Discover the Rest of Reality], Houghton Mifflin Harcourt (2011).

[7] D. Malcara, Ed., [Optical Shop Testing] $3^{\text {rd }}$ Ed., Wiley-Interscience (2007).

[8] Isaac Newton, [Opticks or, a Treatise of the Reflections, Refractions, Inflections, and Colours of Light], Reprinted, Amazon Kindle Edition (2011).

[9] M. Planck, translated by M. Masius, [The Theory of Heat Radiation], now available from Dover and Gutenberg eBook; Blakistons Son \& Co. (1914).

[10] J. Bernstein, P. M. Fishbane \& S. Gasiorowicz []Modern Physics], Prentice Hall (2000).

[11] W. E. Lamb and M. O. Scully Polarization, Matter and Radiation. Jubilee volume in honour of Alfred Kasiler, University of France, Paris, p.363 (1969).

[12] M. D. Crisp and E. T. Jaynes, Phys. Rev. 179 (5), pp.1253-1261 (1969).

[13] C. R. Stroud and E. T. Jaynes, Phys. Rev. A 1 (1), pp.106-121 (1970).

[14] A. O. Barut and J. F. Van Huele, Phys. Rev. A 32 (6), pp. 3187-3195 (1985).

[15] M. O. Scully and M. S. Zubairy, [Quantum Optics], Cambridge University Press (1997).

[16] S.N. Bose. "Plancks Gesetz und Lichtquantenhypothese,” Zeitschrift für Physik 26, 178 (1924).

[17] S.N. Bose. "Wärmegleichgewicht im Strahlungsfeld bei Anwesenheitvon Materie,” Zeitschrift für Physik 27, 384 (1924).

[18] B. Masters, "Satyendra Nath Bose and Bose-Einstein Statistics”, OSA Optics and Photonics News, April (2013).

[19] P. A. M. Dirac, see p.9 in[The Principles of Quantum mechanics], $4^{\text {th }}$ edition, Oxford University Press (1974).

[20] A. Zeilinger et al., "Single- and double-slit diffraction of neutrons," Rev. Mod. Phys., Vol. 60, No. 4, p. 1067, (1988).

[21] C. Roychoudhuri, “Demonstration Using a Fabry-Perot. I. Multiple-Slit Interference”; Am. J. Phys. 43 (12), 1054 (1975)

[22] C. Roychoudhuri, Down load from http://www.natureoflight.org/CP/ ; \#1976.3; "Is Fourier Decomposition Interpretation Applicable to Interference Spectroscopy?” Bol. Inst. Tonantzintla 2 (2), 101 (1976). 